\title{
A modified sliding spectral method and its application to COSMIC radio occultation data
}

\author{
Xian-Sheng Xu ${ }^{1, *}$, Peng Guo ${ }^{2}$ and Zhen-Jie $\mathrm{Hong}^{3}$ \\ ${ }^{1}$ Ningbo Institute of Technology, Zhejiang University, Ningbo, Zhejiang 315000, China. \\ ${ }^{2}$ Shanghai Astronomical Observatory, NAOCAS, Shanghai, PR China. \\ ${ }^{3}$ School of Mathematics 83 Information Science, Wenzhou University, Zhejiang 325000, China. \\ ${ }^{*}$ Corresponding author. e-mail: xuxiansheng666@126.com
}

In the moist lower troposphere, a limitation of the sliding spectral (SS) method is the restriction of the resolution of bending angle profiles because of the atmospheric multipath effect and noise. A modified sliding spectral (MSS) method is proposed in this paper to improve the inversion resolution of SS method in the moist lower troposphere. Simulation results show that the noise in the signal may cause inversion error in the classical SS method. The MSS method can decrease the influence of the noise to some extent. The SS and MSS methods were used to process COSMIC (Constellation Observing System for Meteorology, Ionosphere, and Climate) atmPhs profiles from DOY (day of year) 71-DOY 100 in 2007. The retrieved refractivity profiles were compared with those from the corresponding ECMWF (European Centre for Medium-Range Weather Forecasts) analysis. The results show that the SS method contains systematic positive biases in the $3-10 \mathrm{~km}$ height range and systematic negative biases below $3 \mathrm{~km}$. The MSS method, in comparison to SS method, has decreased the maximum positive bias in the range of 3-10 km height from $0.37 \%$ to $0.23 \%$ in the northern hemisphere, from $1.3 \%$ to $0.25 \%$ in the tropics, and from $0.60 \%$ to $0.35 \%$ in the southern hemisphere. The biases of the MSS method are comparable to those announced for the COSMIC atmPrf profile; the latter is inverted by full spectrum inversion (FSI) method.

\section{Introduction}

Radio occultation (RO) soundings of the Earth's atmosphere allow for observations of refractivity, temperature, pressure and humidity in the neutral atmosphere, and electron density in the ionosphere. The GPS (Global Positioning System) RO technique was essentially validated with the launch of GPS/Meteorology (GPS/MET) mission on April 3, 1995. The receiver used in the Microlab-1 satellite could not track signals in the lower troposphere due to strong attenuations and scintillations, resulting from tropospheric inhomogeneity and atmospheric multipath propagation. On
April 14, 2006, a joint Taiwan-US FORMOSAT-3/ COSMIC (Constellation Observing System for Meteorology, Ionosphere and Climate) mission was launched and is currently providing 1000-2500 RO events per day. Implementation of open-loop (OL) tracking technique in GPS receivers on board COSMIC satellites has two outstanding advantages: (1) OL RO signals can effectively penetrate the atmospheric boundary layer (ABL) without introducing tracking errors, and (2) the ability to receive rising occultations is added, that can double the number of occultations in the troposphere (Sokolovskiy et al. 2006, 2009; Ao et al. 2009). Reduction of both random and systematic temperature errors is found by

Keywords. COSMIC occultation; multipath; inversion; MSS method. 
applying COSMIC profiles to weather forecast and climate analysis assimilation system (Liou et al. 2007; Anthes et al. 2008).

Retrieval of atmospheric parameters from RO data often encounters difficulties in the moist lower troposphere. Under conditions of atmospheric multipath propagation, calculation of bending angle from Doppler frequency shift is usually no longer applicable. Radioholographic $(\mathrm{RH})$ methods to process RO data in atmospheric multipath zones are suggested to improve retrieval accuracy in the moist lower troposphere, such as back propagation (BP) (Kursinski et al. 2000; Gorbunov 2002a), sliding spectral (SS) (Sokolovskiy 2001), canonical transform (CT) (Gorbunov and Kornblueh 2001; Gorbunov 2002b), full spectrum inversion (FSI) (Jensen et al. 2003), CT2 (Gorbunov and Lauritsen 2004), and phase matching (PM) (Jensen et al. 2004).

The SS method uses spectral analysis of the received signals in small sliding apertures. As a function of the impact parameter, the bending angle is computed from the frequency of each spectral maximum and its corresponding position at the aperture center. SS method takes into account the whole spectral content of the signals in the small aperture. Multiple signal classification (MUSIC) technique was used to test SS method by processing 4 GPS/MET occultations (Hocke et al. 1999). By spectral analysis, the contributions from components of surface reflections were detected in 20\%-30\% of CHAMP (Challenging Minisatellite Payload) occultations (Beyerle et al. 2002). Sokolovskiy et al. (2010) thoroughly investigated the bias induced by the noise in $\mathrm{RH}$ methods, and gave a physical explanation. However, false spectral maxima induced by the noise can often result in retrieval errors in SS method. Sorting out the doubtful maxima can improve accuracy of SS method, especially in the lower troposphere (Gorbunov et al. 2000).

In order to identify and decrease the effect of noise in the moist lower troposphere, a modified sliding spectral (MSS) method is developed by using the amplitude and spectral power information of the signal. Both the numerical simulations and statistical comparisons of COSMIC RO refractivity profiles with those from ECMWF (European Centre for Medium-Range Weather Forecasts) analysis are available to validate different inversion methods.

\section{SS method}

The SS method can reconstruct bending angle profile as an unambiguous function of the impact parameter with implementation of the whole GPS signal spectral content in an adopted small aperture, without identification and selection of local spectral maxima (Sokolovskiy 2001).

Assuming that the complex signal $u(t)$ measured along LEO (Low Earth Orbit) trajectory is

$$
u(t)=A(t) \exp [i s(t)]
$$

where $A(t)$ and $s(t)$ are the amplitude and phase of the signal, respectively, $t$ is the time, $i$ denotes imaginary unit. The main trend in the phase can be effectively removed by a reference wave field $s_{\text {mod }}(t)$

$$
v(t)=A(t) \exp \left\{i\left[s(t)-s_{\bmod }(t)\right]\right\}
$$

The reference wave field $s_{\text {mod }}(t)$ can be obtained from cubic spline regression of the phase function in the whole occultation interval. The residual signal $v(t)$ in equation (2) can be decomposed into a set of harmonics in a chosen small aperture $T$

$$
v(t)=\sum_{m=1}^{M_{1}} E_{m} \exp \left\{i s_{m}\right\} \exp \left\{\frac{i 2 \pi m t}{T}\right\}
$$

in which $E_{m}$ and $s_{m}$ are the amplitude and phase of $m$ th component, respectively, $M_{1}$ is the number of harmonics in the aperture.

The bending angle $\alpha_{m}$ and its corresponding impact parameter $a_{m}$ can be calculated from the frequency of each harmonic component and the position of the corresponding aperture center. By sliding the aperture, a set of overlapping physical parameters $\left\{a_{j}, \alpha_{j},\left|E_{j}\right|^{2}\right\}$ is obtained, where $1 \leq j \leq M_{1}\left(M-M_{1}\right), M$ is the number of data in a sounding profile, and $\left|E_{j}\right|^{2}$ denotes the spectral power. The bending angle and corresponding spectral power are then sorted according to increasing impact parameter.

The bending angle is now represented as an unambiguous function of the impact parameter, and can finally be obtained from the sorted array by sliding window averaging weighted by the spectral power

$$
\left\{\begin{array}{l}
\alpha_{l}=\frac{1}{\omega_{l}} \sum_{j=l-L / 2}^{l+L / 2} \alpha_{j}\left|E_{j}\right|^{2} \\
a_{l}=\frac{1}{\omega_{l}} \sum_{j=l-L / 2}^{l+L / 2} a_{j}\left|E_{j}\right|^{2} \\
\omega_{l}=\sum_{j=l-L / 2}^{l+L / 2}\left|E_{j}\right|^{2}
\end{array}\right.
$$

where $L$ (in number of samples) represents window length, $\omega_{l}$ is the sum of the spectral power in the sliding window, and $(L / 2)+1 \leq l \leq M_{1}\left(M-M_{1}\right)-$ $(L / 2)$. The aperture is taken as $0.64 \mathrm{~s}$ (corresponding to 32 samples at $50 \mathrm{~Hz}$ sampling frequency). 
The window length with 300 samples is supposed to provide a reasonable resolution.

In a spherically symmetric atmosphere, the refractive index $n$ as a function of tangent radius $r_{0}$ can be computed from the bending angle $\alpha$ as a function of impact parameter $a$ by using Abel transform (Fjeldbo et al. 1971)

$$
n\left(r_{0}\right)=\exp \left(\frac{1}{\pi} \int_{a}^{\infty} \frac{\alpha(x)}{\sqrt{x^{2}-a^{2}}} \mathrm{~d} x\right) .
$$

The tangent radius $r_{0}$ is related to the impact parameter and the refractive index

$$
r_{0}=\frac{a}{n\left(r_{0}\right)} .
$$

The corresponding Abel integral is:

$$
\alpha(a)=2 a \int_{r_{0}}^{\infty} \frac{1}{\sqrt{n^{2} r^{2}-a^{2}}} \frac{\mathrm{d} \ln n}{\mathrm{~d} r} \mathrm{~d} r .
$$

The refractivity can be written as:

$$
N=(n-1) \cdot 10^{6} \text {. }
$$

The noise in the moist lower troposphere may result in false spectral maxima which degrade the accuracy of the bending angle profile computed by equation (4).

\section{MSS method}

The whole spectral content of RO signal is implemented in the classical SS method, without consideration for detection and removal of the noise. In the moist lower troposphere, due to the noise, the accuracy of the bending angle profile reconstructed by SS method is slightly degraded. The MSS method is designed to reduce the noise influence by using the signal amplitude and spectral power information.

\subsection{Signal reconstruction}

The amplitude of RO signal changes slowly and stably in the single-path area. In the moist lower troposphere, the signal changes rapidly and attenuates severely due to the atmospheric multipath propagation and noise. A possible modification of SS method is to exclude the noise by introducing a criterion function related to amplitude analysis.

The criterion function $\varepsilon(t)$ is related to the signal amplitude $A(t)$ as:

$$
\varepsilon(t)= \begin{cases}\mu_{1} \times\left(1-\frac{A(t)}{A_{\max } / \mu_{2}}\right), & A(t)<\frac{A_{\max }}{\mu_{2}} \\ 0, & A(t) \geq \frac{A_{\max }}{\mu_{2}}\end{cases}
$$

The parameter $A_{\max }$ represents the maximum amplitude in an occultation event. Tunable parameters $\mu_{1}$ and $\mu_{2}$ in equation (8) determine the maximum percent and the start time for excluding the noise, respectively. The criterion function $\varepsilon(t)$ is proportional to $\mu_{1}$ and increases with decreasing amplitude in the amplitude region $A(t)<A_{\max } / \mu_{2}$. Reliable results can be obtained when $\mu_{1} \in[10 \%, 40 \%]$ and $\mu_{2} \in[2,6]$. The sensitivity of inversion results to $\mu_{1}$ and $\mu_{2}$ is discussed based on COSMIC data and ECMWF analysis in section 5.2. The parameters $\mu_{1}=20 \%$ and $\mu_{2}=2$ are adopted in this paper.

The noise in an aperture can be assumed as:

$$
\left|E_{m}\right|^{2}<\varepsilon(t)\left|E_{\max }\right|^{2}
$$

in which $\left|E_{m}\right|^{2}$ denotes the spectral power as defined in equation (3), and $\left|E_{\max }\right|^{2}$ represents the maximum of its spectral power in the aperture. Harmonic with smaller amplitude can be considered as the noise and removed through equation (9). As seen in equation (9), the criterion function $\varepsilon(t)$ determines noise level which should be excluded in the small aperture $T$. Generally, the signal is not fully separable from the noise in both time and frequency domain.

In order to improve the resolution of the bending angle profile, the altitude interval is recommended as the window length instead of the sampling number; which means $j \in\left\{m \mid a_{l} \leq a_{m}<\right.$ $\left.a_{l}+d\right\}$ holds in equation (4). Here $d$ (in length) represents window length. Number of samples in the altitude interval $d$ can be counted and used for sliding window averaging in equation (4). A window length $d=100 \mathrm{~m}$ might provide a suitable and stable result in both numerical simulations (section 4.2) and statistical comparisons (section 5.3).

\subsection{Signal truncation}

Below $3 \mathrm{~km}$ height, equation (9) cannot yet effectively remove all the noise, because of the low signal-to-noise ratio (SNR) in the moist lower troposphere. A simple approach is to adequately truncate the corrupted signal below a certain truncation height (or the corresponding impact parameter) by spectral power analysis. This process can be summarized as follows:

(a) to estimate sums $\omega(a)$ of the spectral powers in the sliding windows using equation (4);

(b) to compute the mean value of the sums of the spectral powers $\overline{\omega(a)}$ from impact height 20-60 km, where the RO signals are fairly stable; 
(c) to exclude the bending angles and corresponding impact parameters with $\omega(a)<\mu_{3} \overline{\omega(a)}$ below impact height $20 \mathrm{~km}$.

The impact height is defined as impact parameter minus the local curvature radius of the Earth. The parameter $\mu_{3}=10 \%$ is adopted in this paper. The above process can find the truncation height and remove the interference signals in the moist lower troposphere. Figure 1 depicts the sums of the spectral powers from a simulated profile (see section 4), in which solid line represents $\omega(a)$, dash-dot line represents $\overline{\omega(a)}$, dash line represents $\mu_{3} \overline{\omega(a)}$. It can be found in figure 1 that the data with relatively low SNR can be simply truncated.

\section{Simulation results}

The simulated signals are generated from a solution of the parabolic approximation of the Helmholtz wave equation computed by the multiple phase screens (MPS) technique. SS, MSS, and FSI methods are applied to the simulated signals with multipath and noise, the retrieved bending angle profiles are compared with those of the direct Abel integral.

\subsection{Simulation of $R O$ signals with atmospheric multipath}

Simulation of RO signals is performed by EGOPS (End-to-End Generic Occultation Performance Simulation and Processing System) software based

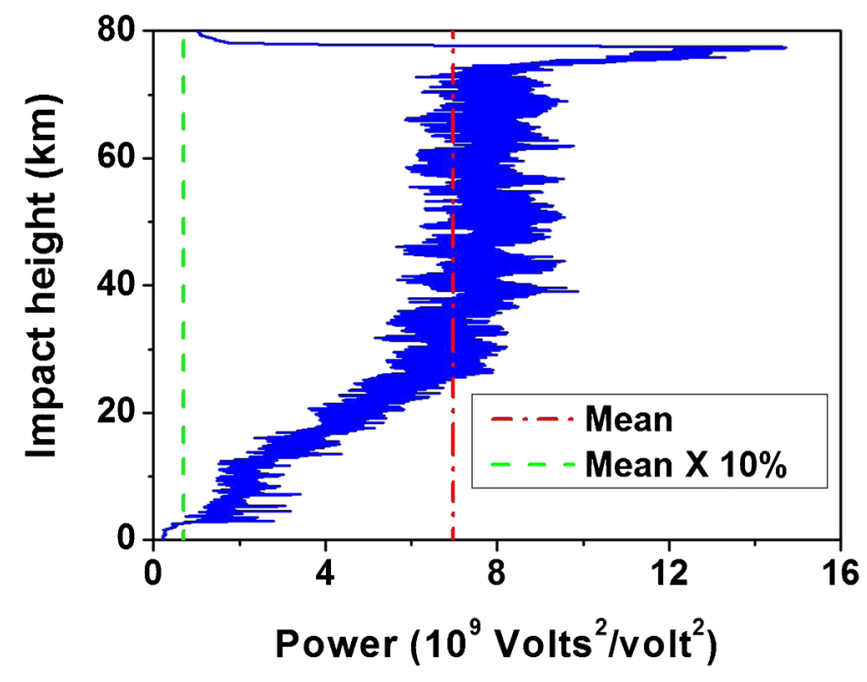

Figure 1. Signal truncation. Solid line represents the sums of the spectral powers, dash-dot line and dash line represent the mean values and their $10 \%$ level of the sums of the spectral powers from 20 to $60 \mathrm{~km}$, respectively. on an ideal atmosphere refractivity profile (Jensen et al. 2003)

$$
N(h)=315 \exp \left[\frac{-h}{7.35 \mathrm{~km}}\right]+10 \exp \left[\frac{-(h-5 \mathrm{~km})^{2}}{0.05 \mathrm{~km}^{2}}\right]
$$

The above profile represents an exponential refractivity with a Gaussian shaped bump. The bump in the second term on the right side of equation (10) is used to illustrate the atmospheric multipath effect.

Under the atmospheric multipath assumption with equation (10), the atmospheric propagation of RO signals is simulated by MPS technique (Sokolovskiy 2001). The distance constant from the first phase screen to the last one is taken to be about $2587 \mathrm{~km}$, the distances between adjacent screens are uniformly taken as $2 \mathrm{~km}$ in simulation experiments. The total number of the phase screens is about 1300 .

For illustrating the multipath effect in the troposphere, the signals with multipath effect were simulated with equation (10). The phase and amplitude of the signal are shown in figure 2(a and $\mathrm{b}$ ). The horizontal axis represents occultation time, and the vertical axes represent the phase and amplitude of the signal, respectively. Attenuations and fluctuations of the amplitude from 42 to $52 \mathrm{~s}$, which are caused by multipath effect, can be found in figure 2(b). The signal without multipath effect is shown in figure $2(\mathrm{c}$ and $\mathrm{d})$, for comparison.

\subsection{Inversion results}

To assess the performance of different retrieval methods, the signal is simulated without and with the noise under multipath condition. In the noisefree scenario, the bending angle profiles reconstructed from the simulated signals by the three methods are compared with those from the direct Abel integral, respectively. In figure 3(a), the bending angle profiles of SS (triangle line), MSS (square line), and FSI (circle line) methods are very close to those from Abel integral (star line) in the single-path region (above $7 \mathrm{~km}$ and below $5 \mathrm{~km}$ impact height). A fine agreement can be found between FSI method and Abel integral from 5 to 7 $\mathrm{km}$, demonstrating the ability of the FSI method to invert bending angle in multipath region. As compared to FSI method, both SS and MSS methods result in retrieval errors in multipath region.

In order to investigate the influence of the noise on the bending angle profiles, we add Gaussian noise with standard deviation of $15 \mathrm{~mm}$ to the phase delay of the simulated signal, and repeat the retrievals (Lohmann 2006). The results in 
figure 3(b) show that SS method is affected by the noise because of false spectral maximum detection. The accuracy of FSI method is also degraded to some extent, especially below $5 \mathrm{~km}$ impact height. This is probably caused by the noise and Gibb's phenomenon due to the finite duration of the received signal (Lohmann et al. 2003). MSS method can retain the accuracy in a noisy environment, because it decreases the influence of the noise through the criterion function equation (9). The comparison results validate that MSS method is less sensitive to the noise than SS and FSI methods.

\section{COSMIC data process}

The accuracy of different methods can be evaluated based on COSMIC data and ECMWF analysis. Refractivity profiles inverted by SS, MSS, and FSI methods from COSMIC data can be compared with those from the corresponding ECMWF analysis. The inversion results of different methods (SS, MSS, and FSI) can be statistically analyzed and compared.

\subsection{COSMIC case study}

Four COSMIC atmPhs profiles, taken from tropical region, on DOY (day of year) 71 and DOY 72 in 2007 are used to describe the verification and comparison among different methods. The results are shown in figure 4.

The retrieved refractivity profiles from atmPhs data by SS, MSS, and FSI methods are compared with those from the corresponding ECMWF analysis; and the comparisons show that SS method results in retrieval error below $8 \mathrm{~km}$ in figure $4(\mathrm{a}-\mathrm{b})$. MSS method can reduce the retrieval error to some extent through the criterion function. In figure $4(\mathrm{c}-\mathrm{d})$, differences are exhibited between the refractivity profiles retrieved by SS method and those from ECMWF analysis below $2 \mathrm{~km}$. In MSS method, some corrupted data below the truncation height of $2 \mathrm{~km}$ are simply truncated by the spectral power information of the signal. Relatively small refractivity differences are found between MSS and FSI methods.

Additionally, MSS method could reach an appropriate retrieval height of refractivity profile. The truncation height of MSS method is generally greater than that of SS method because MSS method performs a signal truncation to the signal. The FSI method has the highest truncation height among the three methods, which might be related to the strict truncation scheme used in FSI method. The four COSMIC examples presented above are taken from tropical region, and have relatively complicated refractivity structure in the lower troposphere. Actually, over $80 \%$ of COSMIC OL profiles retrieved by SS, MSS, and FSI methods
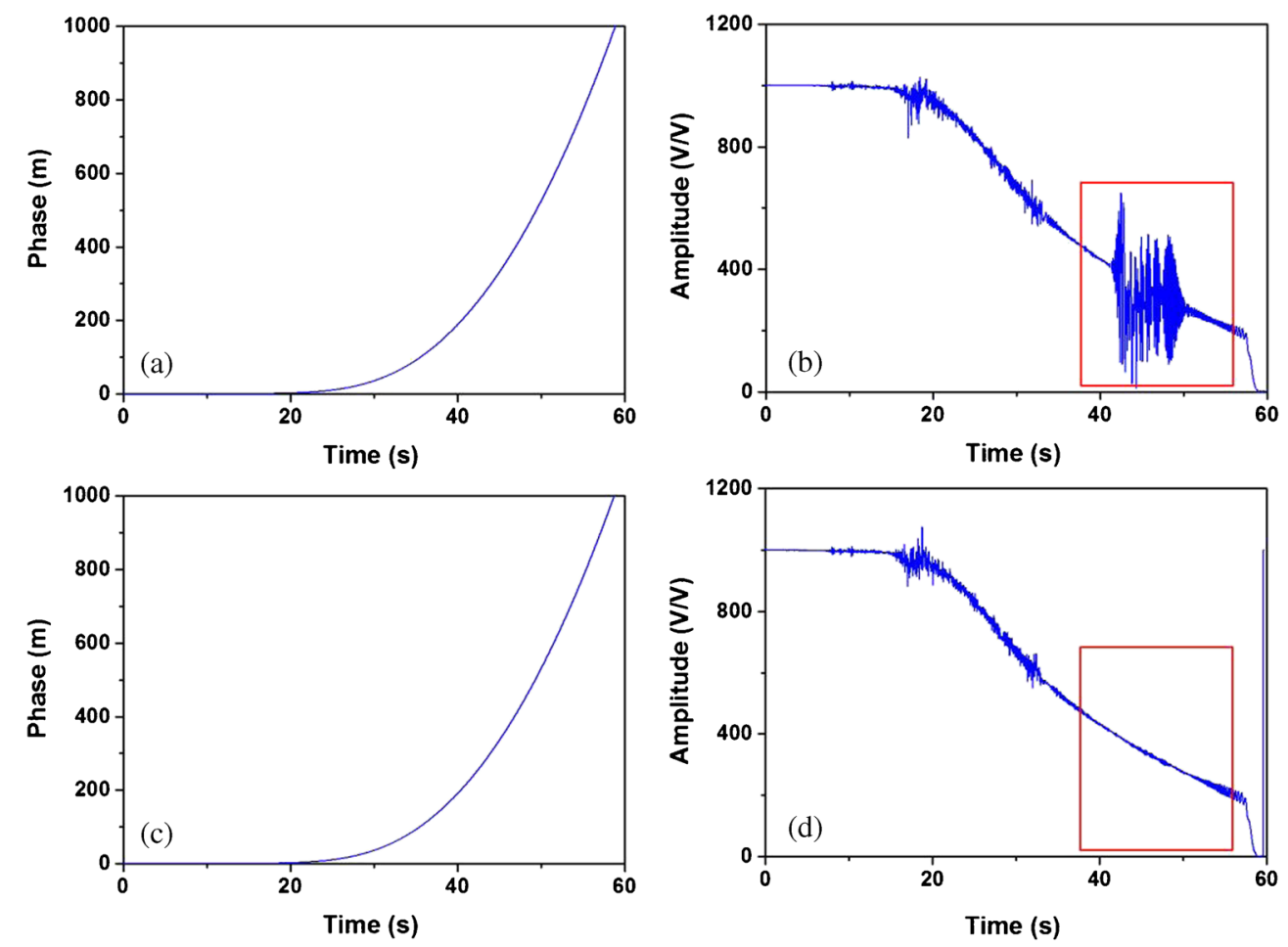

Figure 2. The phase and amplitude of the simulated signals with and without multipath effect: (a-b) with multipath effect; and $(\mathbf{c}-\mathbf{d})$ without multipath effect. 

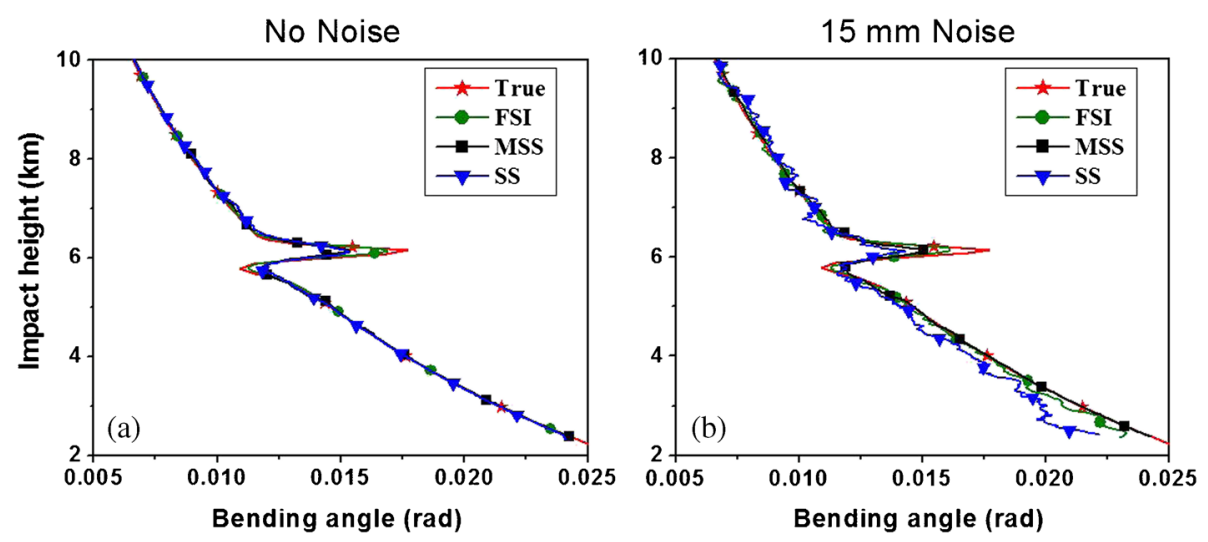

Figure 3. Comparisons of the bending angle profiles reconstructed by SS, MSS and FSI methods with those from Abel integral (taken as the true values): (a) no noise; (b) Gaussian noise with standard deviation of $15 \mathrm{~mm}$. $\rightarrow-$ represents SS method, $\rightarrow$ represents MSS methods, $\rightarrow$ represents FSI method and $₫$ represents Abel integral.

can reach below $2 \mathrm{~km}$ height in the tropics, as seen in section 5.3.

\subsection{Sensitivity analysis}

In this section, we investigate the sensitivity of inversion results to the tunable parameters $\mu_{1}$ and $\mu_{2}$ in MSS method. Figure 5 shows the mean fractional differences (biases) between COSMIC refractivity profiles retrieved by MSS method with different tunable parameters and those of ECMWF analysis for 1047 occultations on DOY 71 in 2007.

The fractional difference of refractivity $\Delta_{f} N$ between COSMIC occultation and ECMWF analysis is defined as:

$$
\Delta_{f} N=\frac{N_{\mathrm{COSMIC}}-N_{\mathrm{ECMWF}}}{\bar{N}}
$$

where $N_{\text {COSMIC }}$ and $N_{\text {ECMwF }}$ represent the refractivity derived from COSMIC occultation and ECMWF analysis, respectively. The symbol $\bar{N}$ denotes the average between $N_{\text {Cosmic }}$ and $N_{\text {ECMWF }}$.

Figure 5(a) shows the mean values of fractional difference in refractivity for different $\mu_{1}$ and fixed $\mu_{2}=2$. Figure 5(b) shows the results for fixed $\mu_{1}=20 \%$ and different $\mu_{2}$. Different tunable parameters result in different inversion biases. Increasing $\mu_{1}$ from $10 \%$ to $40 \%$ results in decrease between 1.5 and $7 \mathrm{~km}$ height and increase below 1.5 $\mathrm{km}$ in the mean fractional difference. It is seen that the sensitivity of results to $\mu_{1}$ is not very strong, while the sensitivity to $\mu_{2}$ is stronger. In this paper, we choose $\mu_{1}=20 \%$ and $\mu_{2}=2$.

\subsection{Statistical comparisons}

Both SS and MSS methods are applied to about 86,880 COSMIC atmPhs profiles from DOY 71 to DOY 100 in 2007, respectively. Figure 6 represents the statistical comparisons of refractivity derived by SS and MSS methods, as well as atmPrf profiles, with those from ECMWF analysis. The atmPrf profiles are inverted by FSI method (Jensen et al. 2003). Both atmPhs and atmPrf profiles can be downloaded from COSMIC Data Analysis and Archive Center (CDAAC) station (http:// cosmic-io.cosmic.ucar.edu/cdaac/index.html).

Figure 6 shows the statistical comparisons of the refractivity in three regions: the northern hemisphere $\left(30^{\circ}-90^{\circ} \mathrm{N}\right)$ (the first row), the tropics $\left(30^{\circ} \mathrm{S}\right.$ to $30^{\circ} \mathrm{N}$ ) (the second row), and the southern hemisphere $\left(30^{\circ}-90^{\circ} \mathrm{S}\right)$ (the last row). The first column of figure 6 is the mean value of the fractional difference of refractivity $\Delta_{f} N$ between COSMIC occultation and ECMWF analysis; the second is their standard deviations; and the third is the collocated number of occultation. The altitude interval is taken as $0.1 \mathrm{~km}$ for the refractivity interpolation. Before statistical comparison, the sample with the fractional difference of refractivity larger than $10 \%$ is considered as an outlier, and excluded from the dataset.

Also, it can be seen in figure 6 that the MSS method has smaller biases than the SS method in the three latitudinal regions. In comparison with ECMWF analysis, small differences in biases of SS and MSS methods are exhibited in the altitude interval of $10-15 \mathrm{~km}$, which is normally corresponding to the single-path area. Systematic positive refractivity biases are exhibited in SS method in the $3-10 \mathrm{~km}$ height range, with the maximum approaching about $0.37 \%$ in the northern hemisphere, about $1.3 \%$ in the tropics, and about $0.60 \%$ in the southern hemisphere. This can primarily be explained by the noise of RO signal in the moist lower troposphere. The MSS method is less sensitive to noise than the SS method in the lower troposphere. The MSS method reduces the maximum 

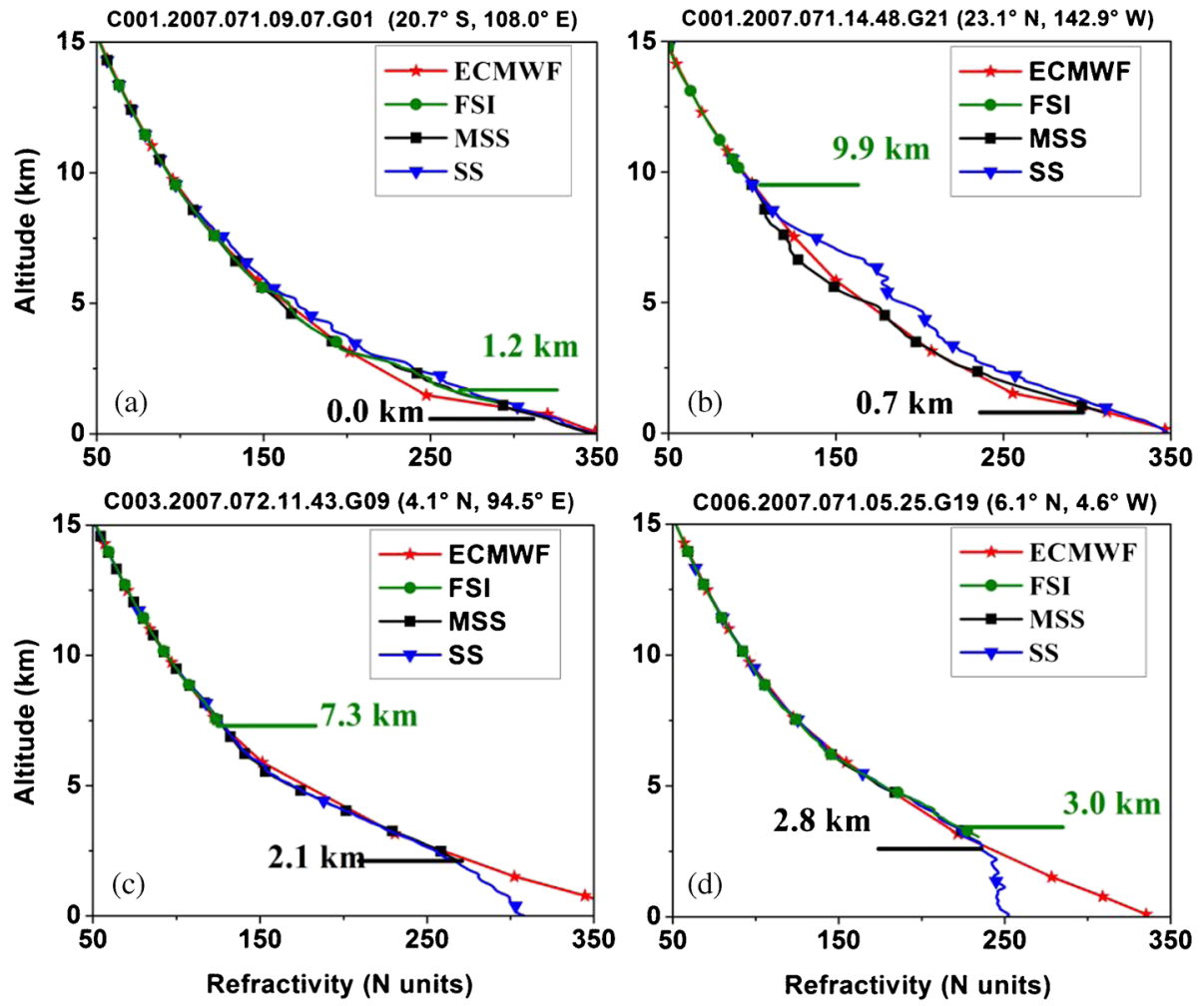

Figure 4. Refractivity comparisons for four COSMIC occultation examples. $\rightarrow$ represents SS method, $\rightarrow-$ represents MSS method, $\rightarrow$ represents FSI method and $\rightarrow$ represents ECMWF analysis.
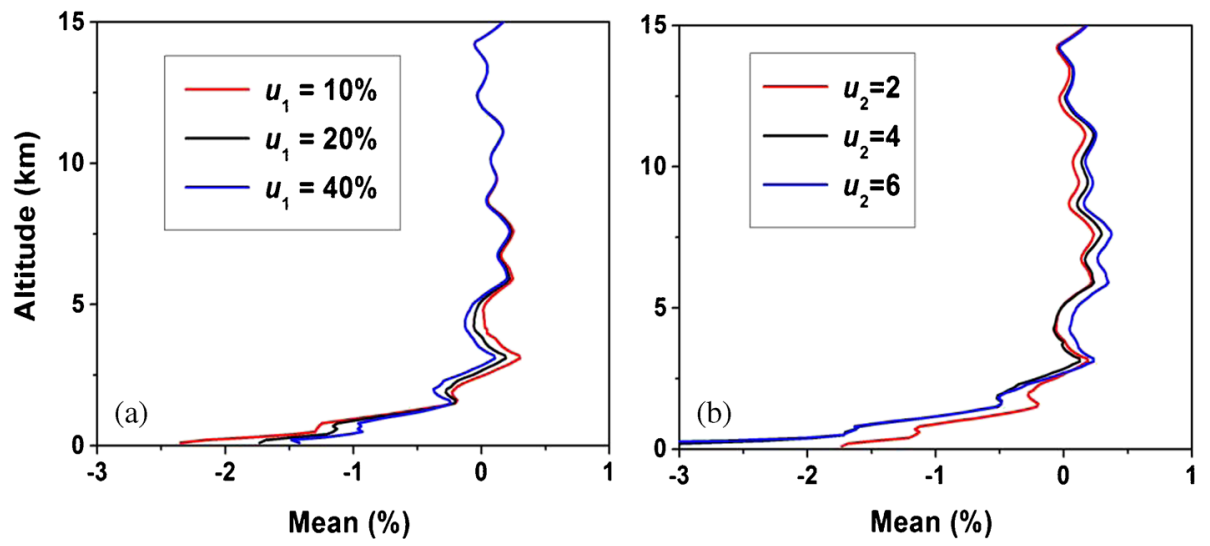

Figure 5. Mean fractional differences between COSMIC refractivities retrieved with different tunable parameters in MSS method and ECMWF refractivities on DOY 71 in 2007. (a) Different $\mu_{1}$ and fixed $\mu_{2}=2$, (b) fixed $\mu_{1}=20 \%$ and different $\mu_{2}$.

positive bias to $0.23 \%$ in the northern hemisphere, $0.25 \%$ in the tropics, and $0.35 \%$ in the southern hemisphere. Below $3 \mathrm{~km}$, systematic negative refractivity biases, presumably caused by atmospheric multipath effect, are verified by both SS and MSS methods. The biases of the MSS method are smaller than those of the SS method.

As can be seen in figure 6 , in both MSS and FSI methods, negative refractivity biases are noticeable in the moist lower tropical troposphere, with a magnitude approaching $2.5 \%$ near the surface
(Ao et al. 2003). These biases are less than $0.7 \%$ in the northern hemisphere and less than $1 \%$ in the southern hemisphere. The performance of the SS method is worse than that of MSS and FSI methods. The negative refractivity bias is mainly related to the global distribution of humidity in the moist lower troposphere.

Small differences in the standard deviations of fractional refractivity difference can be found among SS, MSS, and FSI methods above $2 \mathrm{~km}$ height. The standard deviations of the three 
methods are less than $1 \%$ above $7 \mathrm{~km}$. At about $2 \mathrm{~km}$ height, the standard deviations increase to $1.7 \%$ in the northern hemisphere, $3.5 \%$ in the tropics, and $2.5 \%$ in the southern hemisphere. Below 2 $\mathrm{km}$, SS method has larger standard deviation than MSS and FSI methods, because SS method might be affected by the noise and multipath effect in the lower troposphere.

As seen in figure 6, SS, MSS, and FSI methods can retrieve most occultation profiles above $5 \mathrm{~km}$. The occultation number drops significantly below about $5 \mathrm{~km}$. In the tropics, the percent of retrieved occultation profiles at $2 \mathrm{~km}$ height is $90.2 \%$ for SS method, $87.3 \%$ for MSS method, and $78.5 \%$ for FSI method, respectively. This is mainly related to the different signal truncation schemes used in different methods. In the northern and southern hemispheres, relatively small differences in occultation number can be noticed among the three methods.

In order to compare the penetration ability between the MSS and FSI methods, an investigation on the truncation height of the MSS and FSI methods is made based on the COSMIC data on DOYs 71-100, as seen in figure 7. Figure 7( $\mathrm{a}$ and b) represents the truncation heights of the MSS and FSI methods, respectively. The mean value of the

Northern hemisphere $\left(30^{\circ} \mathrm{N}\right.$ to $\left.90^{\circ} \mathrm{N}\right)$
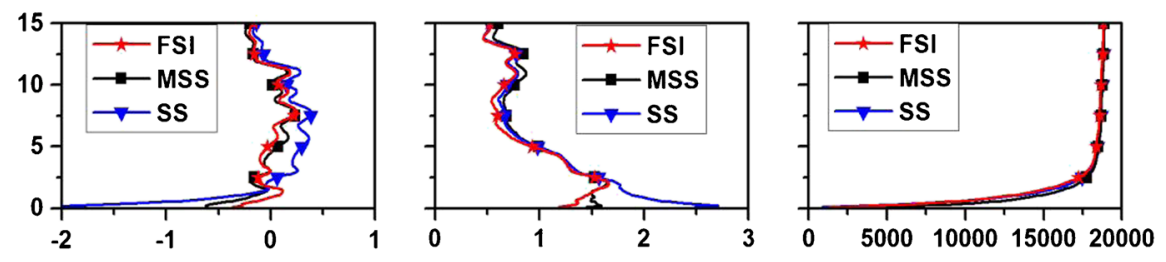

Tropics $\left(30^{\circ} \mathrm{S}\right.$ to $\left.30^{\circ} \mathrm{N}\right)$
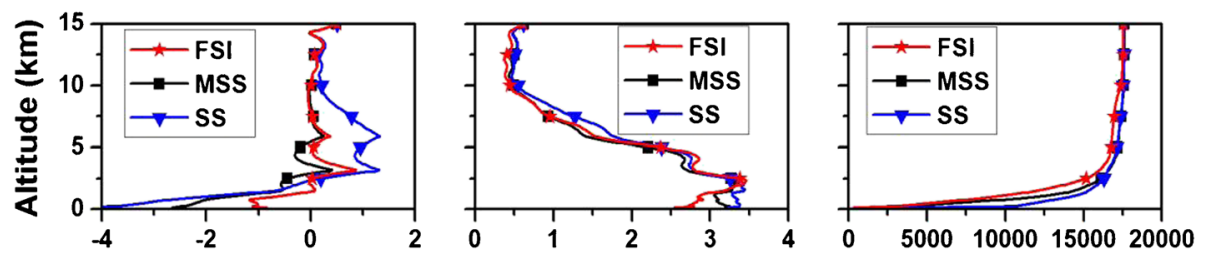

Southern hemisphere $\left(30^{\circ} \mathrm{S}\right.$ to $\left.90^{\circ} \mathrm{S}\right)$
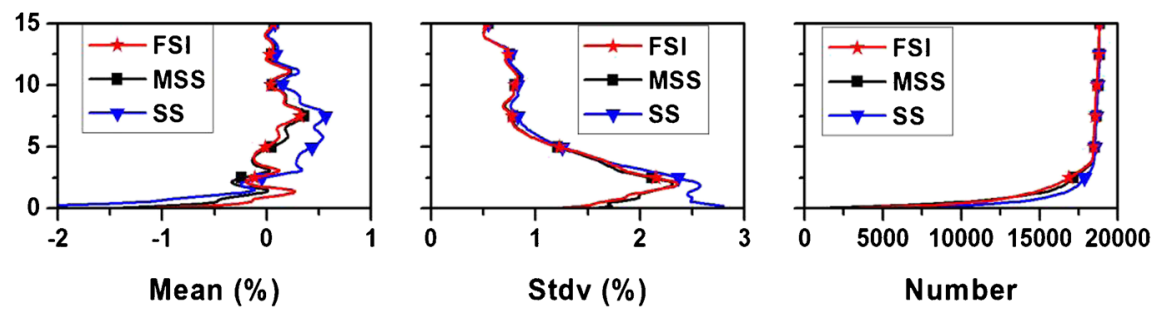

Figure 6. Fractional differences of refractivity between COSMIC occultation and ECMWF analysis for the northern hemisphere, the tropics and the southern hemisphere on DOYs $71-100$ in 2007. \# represents the results from FSI method (atmPrf).
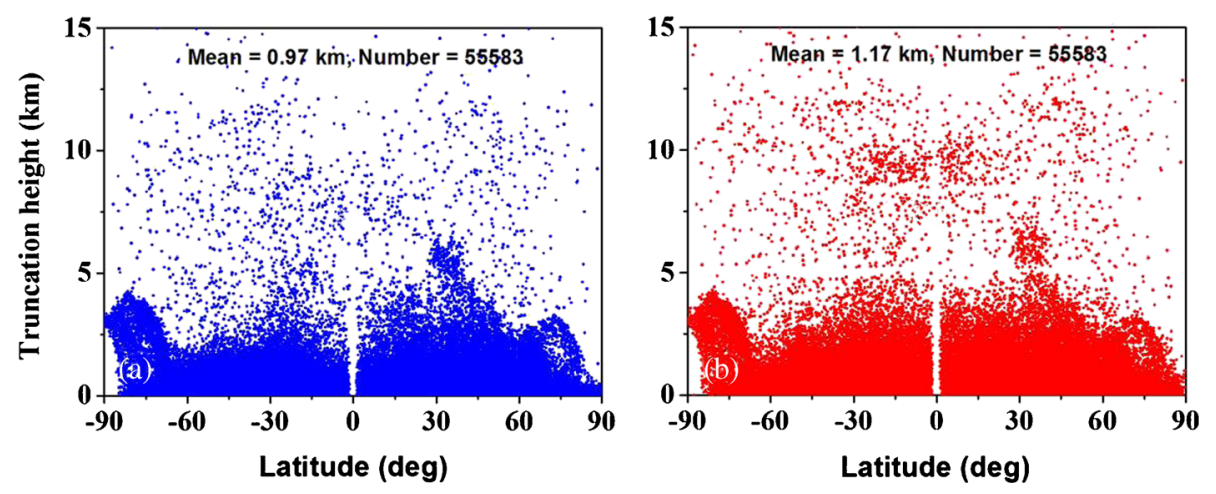

Figure 7. The latitudinal distribution of truncation heights for the MSS and FSI methods based on COSMIC data on DOYs 71-100 in 2007: (a) MSS method and (b) FSI method. 
truncation height of the MSS method is relatively smaller than that of the FSI method. The percentage of occultation that can reach below $1 \mathrm{~km}$ is $70 \%$ for the MSS method, and $63 \%$ for the FSI method. The truncation ability of the MSS method is relatively better than that of the FSI method.

\section{Summary}

A modified sliding spectral (MSS) method is developed in this paper. MSS method can identify and sort out the false spectral maxima in the signal, and adequately truncate the corrupted signal below a certain truncation height. MSS method can decrease the influence of the noise, to some extent, by amplitude and spectral power information of RO signals in both numerical simulations and statistical comparisons. MSS method is superior to SS method in both noise reduction and vertical resolution.

Atmospheric propagation of GPS RO signals is simulated in this paper by MPS technique under atmospheric multipath conditions. Under atmospheric assumptions with noise and multipath, the simulated signals are retrieved by SS, MSS, and FSI methods. Comparisons between bending angle profiles derived by different methods and those from direct Abel integral indicate that FSI method has the best performance in multipath region. The noise in the signal can lead to a degradation of retrieval accuracy in both SS and FSI methods. MSS method is relatively less sensitive to the noise than SS and FSI methods.

Four COSMIC occultation examples, taken from the tropical region, are used to verify the performance of different methods. Comparisons between refractivity profiles retrieved from COSMIC data and those of ECMWF analysis show that SS method results in retrieval errors in the lower troposphere. Both MSS and FSI methods have a better accuracy than SS method. Small refractivity differences are found between MSS and FSI methods. However, FSI method has a larger truncation height than MSS method for its strict truncation scheme.

About 86,880 COSMIC occultations are retrieved by SS and MSS methods, respectively. Statistical comparisons of derived refractivity by SS and MSS methods, as well as COSMIC atmPrf profiles, with those from ECMWF analysis, show that SS method contains systematic biases in the moist lower troposphere. MSS method can decrease influence of the noise in the signal, to some extent, and improve the accuracy of SS method. The systematic bias comparisons of refractivity have proved that the accuracy of MSS method is comparable with that of FSI method. Thus, MSS method can be used as a reference method. Further studies or investigations are needed to broaden the application of $\mathrm{RH}$ methods to data analysis in complicated environment.

\section{Acknowledgements}

The authors are grateful to University Corporation for Atmospheric Research (UCAR) for access to COSMIC RO data and ECMWF analysis data. Thanks are also due to Wegener Centre and University of Graz for providing the EGOPS software. This research is supported by the National Natural Science Foundation of China (11273047, 41305016), the Natural Science Foundation of Zhenjiang province (LQ13D050002) and the Natural Science Foundation of Ningbo (2014A610156, 2014A610022).

\section{References}

Anthes R A, Bernhardt P A, Chen Y, Cucurull L, Dymond K F, Ector D, Healy S B, Ho S P, Hunt D C, Kuo Y H, Liu H, Manning K, McCormick C, Meehan T K, Randel W J, Rocken C, Schreiner W S, Sokolovskiy S V, Syndergaard S, Thompson D C, Trenberth K E, Wee T K, Yen N L and Zeng Z 2008 The COSMIC/FORMOSAT-3 mission: Early results; Bull. Am. Meteor. Soc. 89(3) 313-333, doi: 10.1175/BAMS-89-3-313.

Ao C O, Meehan T K, Hajj G A and Mannucci A J 2003 Lower troposphere refractivity bias in GPS occultation retrievals; J. Geophys. Res. 108(D18) 4577, doi: 10.1029/2002JD003216.

Ao C O, Hajj G A, Meehan T K, Dong D, Iijima B A, Mannucci A J and Kursinski E R 2009 Rising and setting GPS occultations by use of open-loop tracking; $J$. Geophys. Res. 114 D04101, doi: 10.1029/2008JD010483.

Beyerle G, Hocke K, Wickert J, Schmidt T, Marquardt C and Reigber C 2002 GPS radio occultations with CHAMP: A radio holographic analysis of GPS signal propagation in the troposphere and surface reflections; J. Geophys. Res. $\mathbf{1 0 7}(\mathbf{D 2 4})$ 4802, doi: 10.1029/2001JD001402.

Fjeldbo G, Kliore A J and Eshleman V R 1971 The neutral atomosphere of Venus as studied with the Mariner $\mathrm{V}$ radio occultation experiments; Astron. J. 76(2) 123-140.

Gorbunov M E, Gurvich A S and Kornblueh L 2000 Comparative analysis of radioholographic methods of processing radio occultation data; Radio Sci. 35(4) 1025-1034.

Gorbunov M E and Kornblueh L 2001 Analysis and validation of GPS/MET radio occultation data; J. Geophys. Res. 106(D15) 17,161-17,169.

Gorbunov M E 2002a Radio-holographic analysis of Microlab-1 radio occultation data in the lower troposphere; J. Geophys. Res. 107(D12), doi: 10.1029/ 2001JD000889.

Gorbunov M E 2002b Canonical transform method for processing radio occultation data in the lower troposphere; Radio Sci 37(5) 1076, doi: 10.1029/2000RS002592. 
Gorbunov M E and Lauritsen K B 2004 Analysis of wave fields by Fourier integral operators and their application for radio occultations; Radio Sci. 39 RS4010, doi: 10.1029/2003RS002971.

Hocke K, Pavelyev A G, Yakovlev O I, Barthes L and Jakowski N 1999 Radio occultation data analysis by the radioholographic method; J. Atmos. Sol.-Terr. Phys. 61 1169-1177.

Jensen A S, Lohmann M S, Benzon $\mathrm{H} \mathrm{H}$ and Nielsen A S 2003 Full spectrum inversion of radio occultation signals; Radio Sci. 38(3) 1040, doi: 10.1029/ 2002RS002763.

Jensen A S, Lohmann M S, Nielsen A S and Benzon H H 2004 Geometrical optics phase matching of radio occultation signals; Radio Sci. 39 RS3009, doi: 10.1029/2003RS002899.

Kursinski E R, Hajj G A, Leroy S S and Herman B 2000 The GPS radio occultation technique; Terr. Atmos. Oceanic Sci. 11(1) 53-114.

Liou Y A, Pavelyev A G, Liu S F, Pavelyev A A, Yen N, Huang C Y and Fong C J 2007 FORMOSAT-3 GPS radio occultation mission: Preliminary results; IEEE Trans. Geosci. Remote Sens. 45(11) 3813-3826, doi: 10.1109/TGRS.2007.903365.
Lohmann M S, Jensen A S, Benzon H H and Nielsen A S 2003 Radio occultation retrieval of atmospheric absorption based on FSI; Scientific Report 03-20 Danish Meteorological Institute, Copenhagen.

Lohmann M S 2006 Dynamic error estimation for radio occultation bending angles retrieved by the full spectrum inversion technique; Radio Sci. 41 RS5005, doi: 10.1029/2005RS003396.

Sokolovskiy S 2001 Modeling and inverting radio occultation signals in the moist troposphere; Radio Sci. 36(3) 441458.

Sokolovskiy S, Kuo Y H, Rocken C, Schreiner W S, Hunt D and Anthes R A 2006 Monitoring the atmospheric boundary layer by GPS radio occultation signals recorded in the open-loop mode; Geophys. Res. Lett. 33 L12813, doi: 10.1029/2006GL025955.

Sokolovskiy S, Rocken C, Schreiner W, Hunt D and Johnson J 2009 Postprocessing of L1 GPS radio occultation signals recorded in open-loop mode; Radio Sci. 44 RS2002, doi: 10.1029/2008RS003907.

Sokolovskiy S, Rocken C, Schreiner W and Hunt D 2010 On the uncertainty of radio occultation inversions in the lower troposphere; J. Geophys. Res. 115 D22111, doi: 10.1029/2010JD014058. 